\title{
Molecular Identification of Chinese Materia Medica and Its Adulterants Using ITS2 and psbA-trnH Barcodes: A Case Study on Rhizoma Menispermi
}

\author{
Pei Yang1* Xiwen Li1 ${ }^{1}{ }^{*}$, Hong Zhou${ }^{1}$, Hao Hu${ }^{3}$, Hui Zhang4, Wei Sun², Yitao Wang ${ }^{3}$, Hui Yao1\# \\ ${ }^{1}$ Institute of Medicinal Plant Development, Chinese Academy of Medical Sciences and Peking Union Medical \\ College, Beijing, China \\ ${ }^{2}$ Institute of Chinese Materia Medica, China Academy of Chinese Medical Sciences, Beijing, China \\ ${ }^{3}$ State Key Laboratory of Quality Research in Chinese Medicine, Institute of Chinese Medical Sciences, \\ University of Macau, Macau, China \\ ${ }^{4}$ Development Center of Traditional Chinese Medicine and Bioengineering, Changchun University of Chinese \\ Medicine, Changchun, China \\ Email: "scauyaoh@sina.com
}

Received 8 September 2014; revised 16 October 2014; accepted 27 October 2014

Copyright (C) 2014 by authors and Scientific Research Publishing Inc.

This work is licensed under the Creative Commons Attribution International License (CC BY).

http://creativecommons.org/licenses/by/4.0/

(c) (7) Open Access

\section{Abstract}

Rhizoma Menispermi, derived from the rhizoma of Menispermum dauricum DC., is one of the most popular Chinese medicines. However Rhizoma Menispermi is often illegally mixed with other species in the herbal market, including Aristolochia mollissimae Hance, which is toxic to the kidneys and potentially carcinogenic. The use of DNA barcoding to authenticate herbs has improved the management and safety of traditional medicines. In this paper, 49 samples belonging to five species, including 34 samples of $M$. dauricum, from different locations and herb markets in China were collected and identified using DNA barcoding. The sequences of all 34 samples of Rhizoma Menispermi are highly consistent, with only one site variation in internal transcribed spacer 2 (ITS2) of nuclear ribosomal DNA and no variations in the psbA-trnH region. The intra-specific genetic distance is much smaller than inter-specific one. Phylogenetic analysis shows that both sequences allow the successful identification of all species. Nearest distance and BLAST1 methods for the ITS2 and psbA-trnH regions indicate $100 \%$ identification efficiency. Our research shows that DNA barcoding can effectively distinguish Rhizoma Menispermi from its adulterants from both commercial and original samples, which provides a new and reliable way to monitor com-

\footnotetext{
*These two authors contributed equally to this work.

${ }^{\#}$ Corresponding author.
} 
mercial herbs and to manage the modern medicine market.

Keywords

Rhizoma Menispermi, Menispermum dauricum DC., ITS2, psbA-trnH, Identification, Adulterants

\section{Introduction}

Rhizoma Menispermi (Beidougen) is a commonly used traditional Chinese herbal medicine recorded in Chinese Pharmacopoeia as an analgesic and antipyretic drug. Rhizoma Menispermi is derived from the rhizoma of the plant Menispermum dauricum DC. (Menispermaceae), which is widely distributed in North China. Recent studies have shown that the alkaloids from $M$. dauricum have various bioactivities, which include anti-arrhythmic and anti-tumor effects [1]. Zhao et al. stated that the phenolic alkaloids in the rhizome of M. dauricum could protect against brain ischemia injury [2]. In addition, the water-soluble polysaccharides extracted from Rhizoma Menispermi significantly inhibit cell proliferation and DNA synthesis in human ovarian carcinoma SKOV3 cells [3]. Rhizoma Menispermi has become a focus of research because of its newly discovered medicinal properties.

Medication safety is currently a research hot spot, which has become necessary with the increase in market demand for medicinal plants. The rhizome of Aristolochia mollissimae Hance (Aristolochiaceae), an adulterant of Rhizoma Menispermi, shows profound nephrotoxicity and carcinogenicity [4]. Radix et Rhizoma Sophorae Tonkinensis (Shandougen), the root and the rhizome of Sophora tonkinensis Gagnep., is the most common adulterant in the herb market. Several studies have explored traditional methods to distinguish these two medicinal species [5]. In China, M. dauricum is the only species in the genus Menispermum; however, medicinal plants in the same family, such as Stephania tetrandra S. Moore and Cocculus orbiculatus (L.) DC, are commonly interchanged because of their similar morphological characteristics. An accurate identification of these medicinal plants is essential to ensuring the purity and safety of medicines for consumers.

The traditional identification of herbal medicines is mainly based on the morphological characteristics of the source plants, microscopic observations, and the physical form or chemical compositions. These parameters are subjective and can be affected by the external environment, leading to inaccurate classification of herbal authenticity. DNA barcoding has emerged as a cost-effective standard for species identification and has brought a renaissance to the study of taxonomy [6]. DNA barcoding has been proven highly effective in identifying medicinal herbs with high accuracy and reproducibility. The internal transcribed spacer 2 (ITS2) of nuclear ribosomal DNA has been validated as a novel DNA barcode to identify medicinal plant species [7] and is now recommended as the universal DNA barcode for plants [8]. In addition, the China Plant Barcode of Life Group [9] has confirmed that ITS/ITS2 should be incorporated into the core barcode set for seed plants. The chloroplast intergenic spacer, $p s b A$-trnH, has been suggested as a candidate barcode sequence after a large-scale study found high variability and efficiency of detection across a broad range of flowering plants [10]. This region is one of the most variable non-coding regions of the plastid genome in angiosperms [11]. In this study, the ITS2 and psbA-trnH regions were used to distinguish Rhizoma Menispermi from its adulterants. The extraction of DNA from different tissues of herb species may be hampered by different chemical compositions and growing environments. Leaves and other fresh tissues are generally used in published literatures, whereas roots, which have high polysaccharides and polyphenols content, are rarely used. In this study, $M$. dauricum rhizomas were used to verify the use of DNA barcoding in the identification of M. dauricum and its adulterants. Commercial materials were included to evaluate the prospective application of the DNA barcoding method in monitoring crude drugs in the market.

\section{Material and Method}

\subsection{Taxon Sampling}

Forty-nine samples of various herbal species were collected from different locations and markets in China (Table 1). A total of 4 original plant samples and 30 rhizoma samples of $M$. dauricum were collected from main 
Table 1. Detailed information of experimental samples in this study.

\begin{tabular}{|c|c|c|c|c|c|}
\hline \multirow{2}{*}{ Taxon } & \multirow{2}{*}{ Sampling part } & \multirow{2}{*}{ Voucher No. } & \multirow{2}{*}{ Location } & \multicolumn{2}{|c|}{ GenBank Accession NO. } \\
\hline & & & & ITS2 & psbA-trnH \\
\hline Menispermum dauricum & Rhizoma & YC0146MT01 & Chengde, Hebei & KC902480 & КС902468 \\
\hline M. dauricum & Rhizoma & YC0146MT02 & Chengde, Hebei & КС902481 & / \\
\hline M. dauricum & Leaf & YC0146МТ03 & Songfengshan, Heilongjiang & КС902482 & КС902460 \\
\hline M. dauricum & Rhizoma & YC0146МT04 & Songfengshan, Heilongjiang & КС902483 & КС902467 \\
\hline M. dauricum & Rhizoma & YC0146MT05 & Songfengshan, Heilongjiang & КС902484 & КС902466 \\
\hline M. dauricum & Leaf & YC0146MT06 & Chifeng, Inner Mongolia & KC902485 & КС902465 \\
\hline M. dauricum & Rhizoma & YC0146MT07 & Xiaoxinganling, Heilongjiang & КС902486 & КС902464 \\
\hline M. dauricum & Rhizoma & YC0146МT08 & Fushun, Liaoning & KC902487 & КС902463 \\
\hline M. dauricum & Rhizoma & YC0146МТ09 & Wangqing, Jilin & КС902488 & КС902459 \\
\hline M. dauricum & Rhizoma & YC0146MT10 & Wangqing, Jilin & КС902489 & / \\
\hline M. dauricum & Rhizoma & YC0146MT11 & Fengcheng, Liaoning & КС902490 & КС902462 \\
\hline M. dauricum & Rhizoma & YC0146MT12 & Fengcheng, Liaoning & КС902491 & КС902461 \\
\hline M. dauricum & Leaf & YC0146MT13 & Yichun, Heilongjiang & KC902492 & КС902458 \\
\hline M. dauricum & Rhizoma & YC0146MT14 & Fushun, Liaoning & КС902493 & KC902476 \\
\hline M. dauricum & Rhizoma & YC0146MT15 & Xiaoxinganling, Heilongjiang & KC902494 & КC902475 \\
\hline M. dauricum & Rhizoma & YC0146MT16 & Chifeng, Inner Mongolia & КС902495 & КС902474 \\
\hline M. dauricum & Rhizoma & YC0146MT18 & Qingdao, Shandong & КС902496 & КС902473 \\
\hline M. dauricum & Rhizoma & YC0146MT19 & Qingdao, Shandong & КС902497 & КС902472 \\
\hline M. dauricum & Rhizoma & ҮС0146МТ20 & Shangluo, Shaanxi & КС902498 & КС902471 \\
\hline M. dauricum & Rhizoma & YC0146MT21 & Shangluo, Shaanxi & КС902499 & КС902470 \\
\hline M. dauricum & Rhizoma & YC0146MT22 & Ziyang, Sichuan & КС902500 & КС902469 \\
\hline M. dauricum & Rhizoma & YC0146MT23 & Anguo Medicine Market, Hebei & KC902501 & КС902479 \\
\hline M. dauricum & Rhizoma & YC0146MT25 & Bozhou Medicine Market, Anhui & KC902502 & КС902477 \\
\hline M. dauricum & Rhizoma & YC0146MT26 & Bozhou Medicine Market, Anhui & KC902503 & / \\
\hline M. dauricum & Rhizoma & YC0146MT28 & Drug Store I, Beijing & KC902504 & / \\
\hline M. dauricum & Rhizoma & YC0146MT29 & Drug Store I, Beijing & KC902505 & / \\
\hline M. dauricum & Rhizoma & YC0146МТ31 & Drug Store II, Beijing & KC902506 & KC902457 \\
\hline M. dauricum & Rhizoma & YC0146МT32 & Drug Store II, Beijing & KC902507 & КС902456 \\
\hline M. dauricum & Rhizoma & ҮС0146МТ33 & Drug Store III, Beijing & КС902508 & КС902455 \\
\hline M. dauricum & Rhizoma & YC0146МТ34 & Drug Store III, Beijing & KC902509 & / \\
\hline M. dauricum & Rhizoma & YC0146МТ35 & Drug Store IV, Beijing & KC902510 & КС902454 \\
\hline M. dauricum & Rhizoma & YС0146МТ36 & Drug Store IV, Beijing & KC902511 & КС902453 \\
\hline M. dauricum & Rhizoma & YC0146MT37 & Drug Store V, Beijing & KC902512 & / \\
\hline M. dauricum & Leaf & PS0345MT01 & IMPLAD, Beijing & GQ434390 & / \\
\hline
\end{tabular}




\begin{tabular}{|c|c|c|c|c|c|}
\hline \multicolumn{6}{|l|}{ Continued } \\
\hline Sophora tonkinensis & Root & YC0104MT01 & Drug Store II, Beijing & КС902514 & КС902515 \\
\hline So. tonkinensis & Root & YC0104MT02 & Drug Store III, Beijing & KJ766117 & KJ766122 \\
\hline So. tonkinensis & Root & YC0104MT03 & Tianlin County, Guangxi & KJ766116 & KJ766123 \\
\hline So. tonkinensis & Root & YC0104MT04 & Meilin County, Guangxi & KJ766118 & KJ766124 \\
\hline So. tonkinensis & Leaf & PS0228MT01 & Nanning, Guangxi & GQ434351 & GQ434960 \\
\hline Aristolochia mollissima & Leaf & YC0514MT01 & Nanyang, Henan & KJ766113 & KJ766119 \\
\hline A. mollissima & Leaf & YC0514MT02 & Nanyang, Henan & KJ766114 & KJ766120 \\
\hline A. mollissima & Leaf & YC0514MT03 & $\begin{array}{l}\text { South China Botanical } \\
\text { Garden, Guangdong }\end{array}$ & KJ766115 & KJ766121 \\
\hline Stephania tetrandra & Leaf & PS0348MT01 & Danfeng, Shaanxi & / & GQ434988 \\
\hline St. tetrandra & Leaf & PS0348MT02 & Xuancheng, Anhui & I & GQ434989 \\
\hline St. tetrandra & Root & YC0200MT01 & Anguo Medicine Market, Hebei & I & KJ766125 \\
\hline St. tetrandra & Root & YC0200MT02 & Anguo Medicine Market, Hebei & I & KJ766126 \\
\hline Cocculus orbiculatus & Leaf & PS0353MT02 & Xuancheng, Anhui & GQ434395 & GQ434990 \\
\hline C. orbiculatus & Leaf & PS0353MT03 & Chengdu, Sichuan & GQ434396 & GQ434991 \\
\hline C. orbiculatus & Leaf & PS0353MT04 & Putian, Fujian & GQ434397 & GQ434992 \\
\hline
\end{tabular}

“/” means the sample failed to obtain the sequence.

producing areas and different drug stores and markets. Additional 15 samples of species, which were often confused as M. dauricum, were also obtained. All samples were identified by Professor Yulin Lin of Institute of Medicinal Plant Development (IMPLAD), Chinese Academy of Medical Sciences, Peking Union Medical College. The samples were deposited in the herbarium of the institute.

\subsection{DNA Extraction, Amplification and Sequencing}

The surface of the rhizoma samples was first wiped with 75\% ethanol, and the outer epidermis was scraped off. Approximately $40 \mathrm{mg}$ for rhizoma samples and $20 \mathrm{mg}$ for the leaves were used to extract genomic DNA using Bioteke DNA Exaction Kit (Bioteke Co., Beijing). For the rhizome samples, 1\% PVP (Polyvinylpyrrolidone) was mixed before they were crushed. Then the crushed samples with lysate were heated to $56^{\circ} \mathrm{C}$ by water bath for $8 \mathrm{~h}-12 \mathrm{~h}$. The succeeding steps were consistent with those used for the leaf samples. The primers and Polymerase Chain Reaction (PCR) conditions were previously described [7] [12]. All amplified products were bi-directionally sequenced. Data analysis was conducted according to the methods used in previous study [8].

\subsection{Sequence Alignment, Genetic Analysis and Species Identification}

Intra- and inter-specific genetic distances were computed with MEGA 5.0 [13] on the basis of the Kimura 2-Parameter (K2P) model. The sequence lengths and GC contents are listed in Table 2. Phylogenetic trees were constructed according to ITS2 and psbA-trnH regions with the use of the neighbor-joining (NJ) method, with 1000 bootstraps, in MEGA 5.0. Finally, BLAST1 and Nearest Distance methods were applied to species identification, as described in previous studies [7] [14].

\section{Results}

\subsection{PCR, Sequencing Efficiency and Sequence Characteristics}

Most genomic DNA samples showed smearing on agarose gel electrophoresis, which indicated DNA degradation. However, most of the samples were successfully amplified except the ITS2 sequence of $S$. tetrandra, suggesting that amplification of both ITS2 and psbA-trnH was not affected by the degradation of genomic DNA. 
Table 2. Sequence characteristics of ITS2 and psbA-trnH of $M$. dauricum and its adulterants.

\begin{tabular}{ccc}
\hline & ITS2 & psbA-trnH \\
\hline Amplification efficiency of M. dauricum (\%) & 100 & 100 \\
Sequencing efficiency of M. dauricum (\%) & 100 & 76.5 \\
Length of M. dauricum (bp) & 203 & 315 \\
Amplification efficiency of all taxa (\%) & 91.8 & 100 \\
Sequencing efficiency of all taxa (\%) & 91.8 & 83.7 \\
Length of all taxa (bp) & $181-277$ & $215-656$ \\
Aligned length (bp) & 331 & 743 \\
GC content range in M. dauricum (\%) & 52.7 & 24.4 \\
GC content range (mean) in all taxa (\%) & $49.3-74.4$ (62.8) \\
Number (and \%) of variable sites in all taxa & 163 (49.2) & $31.4(26.6)$ \\
\hline
\end{tabular}

The amplification efficiency of $p s b A$-trn $H$ was $100 \%$, whereas the sequencing efficiency of $p s b A-t r n H$ was $76.5 \%$ (Table 2). We failed to obtain the ITS2 sequence for S. tetrandra by using the universal ITS2 primer pairs described by Chen et al. [6]. However, the psbA-trnH region was obtained from all the samples of this species.

The sequence length of ITS2 in $M$. dauricum was 203 bp, with only one variable site observed in 34 sequences. The result of inter-specific sequence alignment showed that the length of ITS2 was 331 bp with 163 bp variable sites (49.2\%). The sequence length of $p s b A-t r n H$ in all $M$. dauricum samples was $315 \mathrm{bp}$, which indicated high consistency. The length of $p s b A$-trnH sequences alignment was $743 \mathrm{bp}$ with high variability (42.9\%) across taxa. The GC contents of ITS2 and $p s b A-t r n H$ of $M$. dauricum were $52.7 \%$ and $24.4 \%$, which averaged $62.8 \%$ and $26.6 \%$, respectively across all taxa (Table 2). One of the adulterants, A. mollissima, had the highest GC content with $74.4 \%$ in the ITS2 locus.

\subsection{Intra- and Inter-Specific Genetic Distances and Species Identification}

The intra- and inter-specific K2P distances of ITS2 and psbA-trnH of M. dauricum and its adulterants are presented in Table 3. All the experimental samples of $M$. dauricum were highly conserved in ITS2 and $p s b A$-trnH regions. The maximum intra-specific distance (0.005 in ITS2) was due to a single C-A variation at the 82 bp site. The inter-specific K2P distances of the two sequences were expectedly higher, with an average of 0.457 and 0.428 for ITS2 and $p s b A$-trnH respectively. NJ trees indicated that both sequences allowed the successful identification of all species; with each separate species grouped into distinct clades (Figure 1 and Figure 2). All the sequences were examined by the Nearest Distance and BLAST1 methods. The successful identification rates of the two regions were $100 \%$ with the two methods.

\section{Discussion}

Fresh leaves have been traditionally used as the raw material for DNA barcoding. Currently, DNA barcoding is used to identify traditional Chinese medicines derived from other source tissues such as Rhizoma et Radix Notopterygii [15], Radix Gentianae Macrophyllae [16], Herba Ephedrae [17], and Flos Lonicerae Japonicae [18]. Chiou et al. [19] described that DNA extracted from various medicinal tissues and processed materials was usually degraded and even contaminated by microorganisms. For root and rhizome medicinal materials, the accumulation of secondary metabolites, such as polysaccharides and polyphenols, can affect the quantity and purity of the extracted DNA and the success of downstream applications, including PCR. The general approaches to improve the quality and quantity of DNA extracted from medicinal parts include extending the water bath time to $8 \mathrm{~h}-12$ $\mathrm{h}$ at $56^{\circ} \mathrm{C}$, increasing the amount of the sample, and simultaneously increasing the use of beta-mercaptoethanol and PVP. In addition, extracting the crushed samples by buffer for two to three times [20] will help to remove the polysaccharides and polyphenols in the medicinal parts of crude drugs. In this study, 36 rhizoma samples were collected, including 16 dried and long-stored rhizoma samples of commercial materials, and successfully 


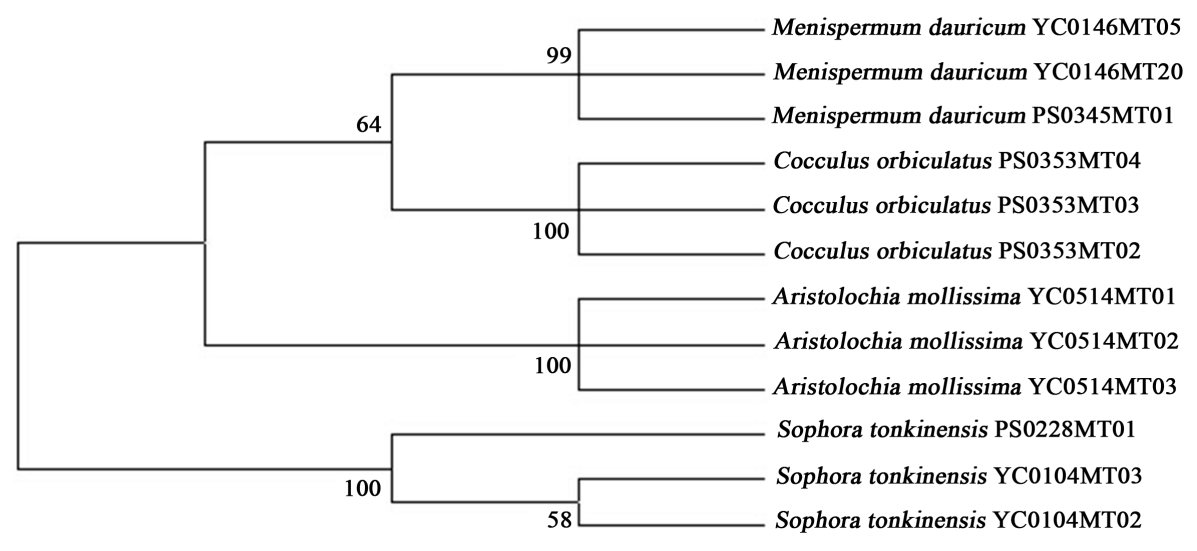

Figure 1. Phylogenetic tree of Rhizoma Menispermi and its adulterants constructed with the representative ITS2 sequences using NJ method. The bootstrap scores (1000 replicates) are shown ( $\geq 50 \%)$ for each branch.

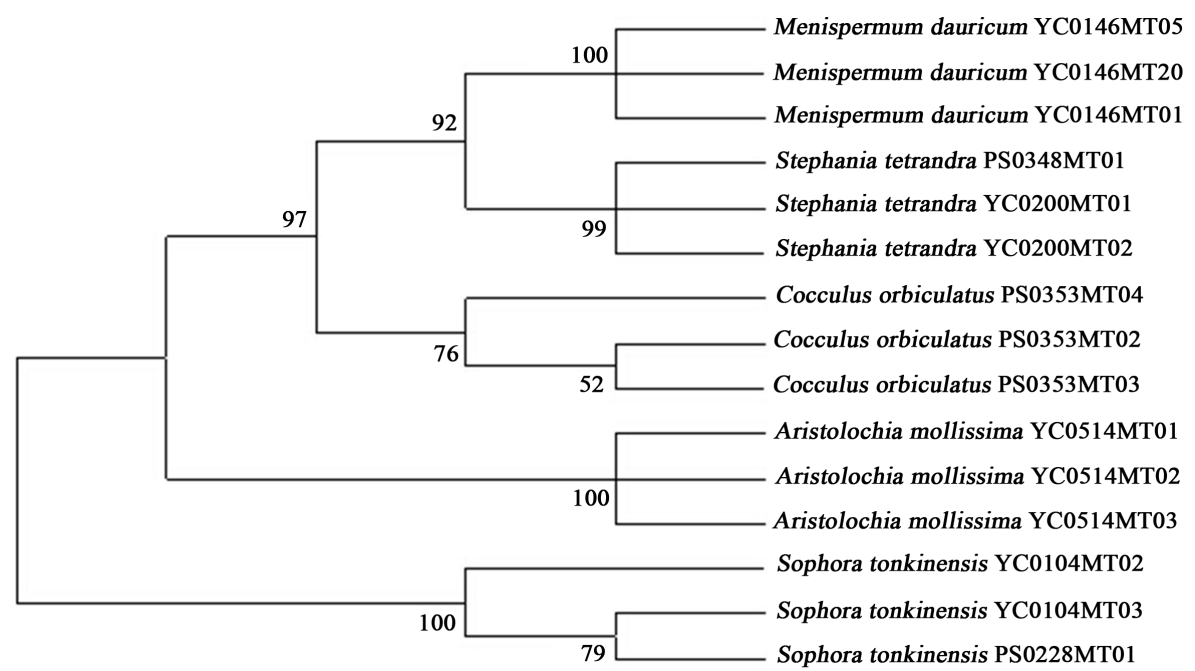

Figure 2. Phylogenetic tree of Rhizoma Menispermi and its adulterants constructed with the representative $p s b A$-trnH sequences using $\mathrm{NJ}$ method. The bootstrap scores (1000 replicates) are shown $(\geq 50 \%)$ for each branch.

Table 3. The K2P genetic distances of ITS2 and psbA-trnH sequences of Rhizoma Menispermi and its adulterants.

\begin{tabular}{ccc}
\hline \multirow{2}{*}{ K2P genetic distances } & \multicolumn{2}{c}{ Range of genetic distances (mean) } \\
\cline { 2 - 3 } & ITS2 & $p s b A-t r n H$ \\
\hline Intra-specific distances of M. dauricum & $0-0.005(0.000)$ & $0(0.000)$ \\
Inter-specific distances between Rhizoma Menispermi and its adulterants & $0.035-0.509(0.457)$ & $0.033-0.624(0.428)$ \\
\hline
\end{tabular}

amplified by PCR. The result showed that the primers used were universal, and the ITS2 and psbA-trnH loci were easy to amplify. Wiping with $75 \%$ ethanol and scraping the outer epidermis helped prevent the risk of soil fungal contamination to a certain extent. No fungal sequence interference was observed during the experiments. $\mathrm{Xu}$ et al. [21] verified the operability of ITS2 locus between $S$. tonkinensis and its adulterants, including $M$. dauricum, using sequences downloaded from GenBank and several plant materials. DNA barcoding could identify fresh original plant samples of $S$. tonkinensis and its adulterants. However, no medicinal material samples were included. In this study, we focused on testing the applicability of DNA barcoding to distinguish medicinal materials. 
New technology contributes to the success of identification methods. In the 2010 edition of Chinese Pharmacopoeia, allele-specific diagnostic PCR was recorded as a new method of identifying Zaocys dhumnades (Cantor). DNA barcoding technology uses universal primers and the same PCR reaction conditions across samples to construct a reference database. These factors increase the value of such a method in practical applications. The Food and Drug Administration approved DNA barcoding for seafood identification [22]. In the contemporary medicine market, this technology will help stop the spread of fake and adulterant materials with similar appearance to true medicines. Kool et al. [23] used root samples sold by herbalists in Marrakech, Morocco, to determine the applicability of DNA barcoding to modern drug markets. The results of their study showed that $r p o C 1$, psbA-trnH, and ITS had high sequencing success rates, and combining all three loci allowed the identification of the majority of the market samples up to the genus level. DNA barcoding was used to successfully detect substitution and contamination in herbal products in North America [24]. These two previous studies indicated that DNA barcoding can be applied to the identification of commercial herbs. In the current study, we collected 16 commercial medicinal materials, including Rhizoma Menispermi, Radix et Rhizome Sophorae Tonkinensis, and Radix Stephaniae Tetrandrae. DNA is expected to show increasing degradation with an increase in storage time in dried commercial root drugs. We show that all the commercial materials were effectively identified, and the results were consistent with the information indicated on the medicinal drug labels. Sequence conservation in commercial medicinal materials and original samples from a single species indicated that DNA barcoding can consistently identify commercialized medicinal plants.

The psbA-trnH region is among the most variable non-coding regions in the chloroplast genome, and this variation means that this intergenic spacer can offer high levels of species discrimination [11]. However, Chase et al. [25] stated that a high rate of insertion/deletion may hamper application to barcoding. Agarose gel electrophoresis demonstrated that both ITS2 and psbA-trnH had high amplification efficiency. However, the sequencing efficiency of psbA-trnH sequences was almost $10 \%$ lower than that of the ITS2 sequences, even if bidirectional sequencing was used. This result was likely due to poly-A and poly-T structures in the psbA-trnH intergenic spacer. When a continuous repeated A or $\mathrm{T}$ structure emerges, the quality of the followed sequence will decrease, so the determination of the complete intergenic spacer is limited. The sequencing efficiency caused a 23.5\% decline compared with the amplification efficiency in M. dauricum. In all samples used in this study, we also observed continuous repeated nucleotide structures belonging to Menispermaceae and S. tonkinensis (Leguminosae).

DNA barcoding technology has contributed to the development of taxonomy [6] and authentication, especially in traditional Chinese herbal medicine. A stable and accurate method is urgently needed for the circulation and standard management of traditional Chinese herbal medicines. This study is the first to use DNA barcoding to authenticate Rhizoma Menispermi (particularly commercial variants) and its adulterants. The stability and accuracy of the two regions (ITS2 and $p s b A-t r n H$ ) were examined. The intra-specific genetic distances and sequence alignment indicated that $M$. dauricum from different production areas had few variable sites in ITS2 and psbA-trnH sequences. The existence of multiple copies of the ITS2 region in plants may affect the stability of the obtained sequences. However, Song et al. [26] showed that despite multiple intra-genomic variants, the use of the major variants alone was sufficient for phylogenetic construction and species determination in most cases. The ITS2 sequences of $M$. dauricum were highly consistent; intra-specific variations were nearly absent. Multi-copies of ITS2 did not affect sequence amplification, sequencing, and analysis in M. dauricum. The high conservation of the ITS2 region was also discovered in Panax ginseng and Panax quinquefolius [27].

\section{Conclusion}

In conclusion, both ITS2 and psbA-trnH can be successfully amplified from commercial and natural samples of the important traditional Chinese medicine, Rhizoma Menispermi and its adulterants. As a result, accurate species identification and validation of product authenticity are facilitated. Compared with the ITS2 regions, the psbA-trnH sequence should not be the first choice for the DNA barcoding of Menispermaceae species on the basis of high poly-N structures and reduced sequencing efficiency. However, in consideration of the results for $S$. tetrandra, psbA-trnH can still be used as a complementary barcode for ITS2 in species identification.

\section{Acknowledgements}

We thank Dr. Jingyuan Song from Institute of Medicinal Plant Development, Chinese Academy of Medical 
Sciences for revising an earlier version of this manuscript. This work was supported by the Program for Changjiang Scholars and Innovative Research Team (No. IRT1150), and National Natural Science Foundation of China (No. 81073001, 81373922).

\section{References}

[1] Shan, B.E., Ren, F.Z., Liang, W.J., Zhang, J., Li, Q.X. and Zeng, Y.P. (2006) Isolation and Purification of Antitumor Component in Rhizoma Menispermi and Analysis of Its Activity. Carcinogenesis, Teratogenesis \& Mutagenesis, 3, No. 007.

[2] Zhao, B., Chen, Y., Sun, X., Zhou, M., Ding, J., Zhan, J.J. and Guo, L.J. (2012) Phenolic Alkaloids from Menispermum dauricum Rhizome Protect against Brain Ischemia Injury via Regulation of GLT-1, EAAC1 and ROS Generation. Molecules, 17, 2725-2737. http://dx.doi.org/10.3390/molecules17032725

[3] Lin, M., Xia, B., Yang, M., Gao, S., Huo, Y. and Lou, G. (2013) Characterization and Antitumor Activities of a Polysaccharide from the Rhizoma of Menispermum dauricum. International Journal of Biological Macromolecules, 53, 7276. http://dx.doi.org/10.1016/j.ijbiomac.2012.11.012

[4] Hoang, M.L., Chen, C.H., Sidorenko, V.S., He, J., Dickman, K.G., Yun, B.H., Moriya, M., Niknafs, N., Douville, C., Karchin, R., Turesky, R.J., Pu, Y.S., Vogelstein, B. and Papadopoulos, N. (2013) Mutational Signature of Aristolochic Acid Exposure as Revealed by Whole-Exome Sequencing. Science Translational Medicine, 5, 197ra102.

[5] Chen, H. (2010) The Difference and the Reasonable Application of Menispermum dauricum and Sophorae tonkinensis. Chinese Medicine Modern Distance Education of China, 8, 60-61.

[6] Miller, S.E. (2007) DNA Barcoding and the Renaissance of Taxonomy. Proceedings of the National Academy of Sciences of the United States of America, 104, 4775-4776. http://dx.doi.org/10.1073/pnas.0700466104

[7] Chen, S.L., Yao, H., Han, J.P., Liu, C., Song, J.Y., Shi, L.C., Zhu, Y.J., Ma, X.Y., Gao, T., Pang, X.H., Luo, K., Li, Y., Li, X.W., Jia, X.C., Lin, Y.L. and Leon, C. (2010) Validation of the ITS2 Region as a Novel DNA Barcode for Identifying Medicinal Plant Species. PLoS One, 5, e8613. http://dx.doi.org/10.1371/journal.pone.0008613

[8] Yao, H., Song, J., Liu, C., Luo, K., Han, J.P., Li, Y., Pang, X.H., Xu, H.X., Zhu, Y.J., Xiao, P.G. and Chen, S.L. (2010) Use of ITS2 Region as the Universal DNA Barcode for Plants and Animals. PLoS One, 5, 13102. http://dx.doi.org/10.1371/journal.pone.0013102

[9] Li, D.Z., Gao, L.M., Li, H.T., Wang, H., Ge, X.J., Liu, J.Q., Chen, Z.D., Zhou, S.L., Chen, S.L., Yang, J.B., Fu, C.X., Zeng, C.X., Yan, H.F., Zhu, Y.J., Sun, Y.S., Chen, S.Y., Zhao, L., Wang, K., Yang, T. and Duan, G.W. (2011) Comparative Analysis of a Large Dataset Indicates That Internal Transcribed Spacer (ITS) Should Be Incorporated into the Core Barcode for Seed Plants. Proceedings of the National Academy of Sciences of the United States of America, 108, 19641-19646. http://dx.doi.org/10.1073/pnas.1104551108

[10] Kress, W.J., Wurdack, K.J., Zimmer, E.A., Weigt, L.A. and Janzen, D.H. (2005) Use of DNA Barcodes to Identify Flowering Plants. Proceedings of the National Academy of Sciences of the United States of America, 102, 8369-8374. http://dx.doi.org/10.1073/pnas.0503123102

[11] Shaw, J., Lickey, E.B., Schilling, E.E. and Small, R.L. (2007) Comparison of Whole Chloroplast Genome Sequences to Choose Noncoding Regions for Phylogenetic Studies in Angiosperms: The Tortoise and the Hare III. American Journal of Botany, 94, 275-288. http://dx.doi.org/10.3732/ajb.94.3.275

[12] White, T.J. (1990) Amplification and Direct Sequencing of Fungal Ribosomal RNA Genes for Phylogenetics. In: PCR Protocols, a Guide to Methods and Applications, 315-322.

[13] Tamura, K., Peterson, D., Peterson, N., Stecher, G., Nei, M. and Kumar, S. (2011) MEGA5: Molecular Evolutionary Genetics Analysis Using Maximum Likelihood, Evolutionary Distance, and Maximum Parsimony Methods. Molecular Biology and Evolution, 28, 2731-2739. http://dx.doi.org/10.1093/molbev/msr121

[14] Ross, H.A., Murugan, S. and Li, W.L. (2008) Testing the Reliability of Genetic Methods of Species Identification via Simulation. Systematic Biology, 57, 216-230. http://dx.doi.org/10.1080/10635150802032990

[15] Xin, T.Y., Yao, H., Luo, K., Xiang, L., Ma, X.C., Han, J.P., Lin, Y.L., Song, J.Y. and Chen, S.L. (2012) Stability and Accuracy of the Identification of Notopterygii Rhizoma et Radix Using the ITS/ITS2 Barcodes. Yao Xue Xue Bao, 47, 1098-1105.

[16] Luo, K., Ma, P., Yao, H., Xin, T.Y., Hu, Y., Zhen, S.H., Huang, L.F., Liu, J. and Song, J.Y. (2012) Identification of Gentianae Macrophyllae Radix Using the ITS2 Barcodes. Yao Хие Хue Bao, 47, 1710-1717.

[17] Pang, X.H., Song, J.Y., Xu, H.B. and Yao, H. (2012) Using ITS2 Barcode to Identify Ephedraeherba. China Journal of Chinese Materia Medica, 37, 1118-1121.

[18] Hou, D.Y., Song, J.Y., Shi, L.C., Ma, X.C., Xin, T.Y., Han, J.P., Xiao, W., Sun, Z.Y., Cheng, R.Y. and Yao, H. (2013) Stability and Accuracy Assessment of Identification of Traditional Chinese Materia Medica Using DNA Barcoding: A 
Case Study on Flos Lonicerae Japonicae. BioMed Research International, 2013, Article ID: 549037.

[19] Chiou, S.J., Yen, J.H., Fang, C.L., Chen, H.L. and Lin, T.Y. (2007) Authentication of Medicinal Herbs Using PCRAmplified ITS2 with Specific Primers. Planta Medica, 73, 1421-1426. http://dx.doi.org/10.1055/s-2007-990227

[20] Li, J.L., Wang, S., Yu, J., Wang, L. and Zhou, S.L. (2013) A Modified CTAB Protocol for Plant DNA Extraction. Chinese Bulletin of Botany, 48, 72-78. http://dx.doi.org/10.3724/SP.J.1259.2013.00072

[21] Xu, X.L., Shi, L.C., Song, J.Y., Han, J.P., Yao, H., Chen, S.L., et al. (2012) Molecular Identification of Sophorae Tonkinensis Radix et Rhizoma Original Plant and Its Adulterants Based on ITS2 DNA Barcode. World Science and Technology/Modernization of Traditional Chinese Medicine and Materia Medica, 14, 1147-1152.

[22] Yancy, H.F., Zemlak, T.S., Mason, J.A., Washington, J.D., Tenge, B.J. and Nguen, N.T. (2008) Potential Use of DNA Barcodes in Regulatory Science: Applications of the Regulatory Fish Encyclopedia. Journal of Food Protection, 71, 210-217.

[23] Kool, A., Boer, H.J., Kruger, A., Rydberg, A., Abbad, A., Bjork, L. and Martin, G. (2012) Molecular Identification of Commercialized Medicinal Plants in Southern Morocco. PLoS One, 7, e39459. http://dx.doi.org/10.1371/journal.pone.0039459

[24] Newmaster, S.G., Grguric, M., Shanmughanandhan, D., Ramalingam, S. and Ragupathy, S. (2013) DNA Barcoding Detects Contamination and Substitution in North American Herbal Products. BMC Medicine, 11, 222. http://dx.doi.org/10.1186/1741-7015-11-222

[25] Chase, M.W., Cowan, R.S., Hollingsworth, P.M., Berg van den, C., Madrinan, S., Petersen, G. and Seberg, O. (2007) A Proposal for a Standardised Protocol to Barcode All Land Plants. Taxon, 56, 295-299.

[26] Song, J.Y., Shi, L.C., Li, D.Z., Sun, Y.Z., Niu, Y.Y., Chen, Z.D., Luo, H.M., Pang, X.H., Sun, Z.Y., Liu, C., Lv, A.P., Deng, Y.P., Larson-Rabin, Z., Wilkinson, M. and Chen, S.L. (2012) Extensive Pyrosequencing Reveals Frequent IntraGenomic Variations of Internal Transcribed Spacer Regions of Nuclear Ribosomal DNA. PLoS One, 7, e43971. http://dx.doi.org/10.1371/journal.pone.0043971

[27] Chen, X.C., Liao, B.S., Song, J.Y., Pang, X.H., Han, J.P. and Chen, S.L. (2013) A Fast SNP Identification and Analysis of Intraspecific Variation in the Medicinal Panax Species Based on DNA Barcoding. Gene, 530, 39-43.

http://dx.doi.org/10.1016/j.gene.2013.07.097 
Scientific Research Publishing (SCIRP) is one of the largest Open Access journal publishers. It is currently publishing more than 200 open access, online, peer-reviewed journals covering a wide range of academic disciplines. SCIRP serves the worldwide academic communities and contributes to the progress and application of science with its publication.

Other selected journals from SCIRP are listed as below. Submit your manuscript to us via either submit@scirp.org or Online Submission Portal.
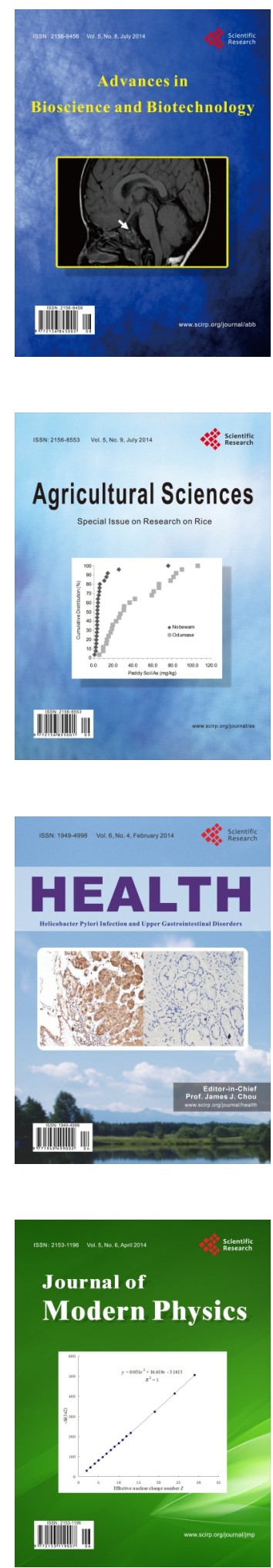
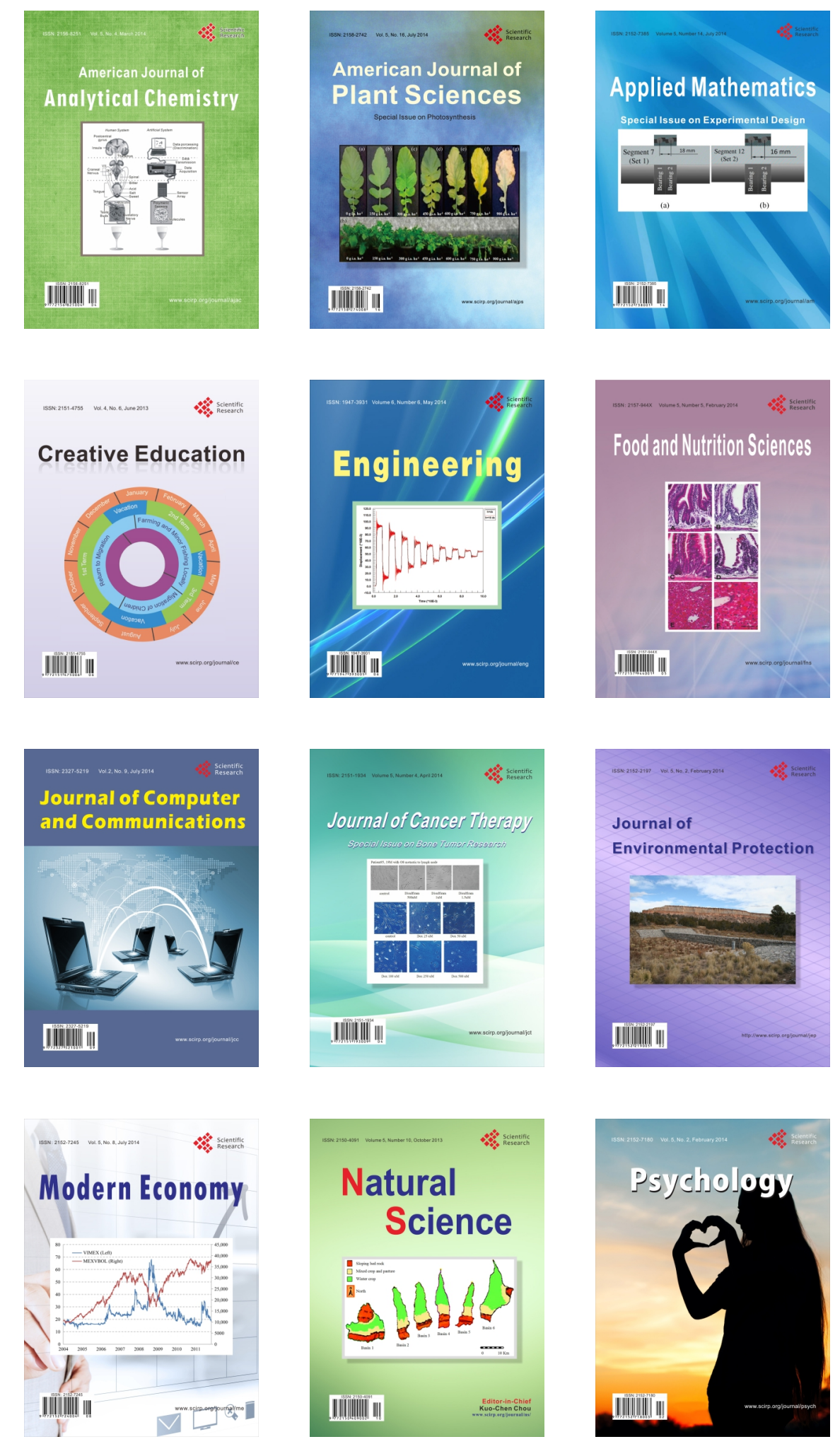